\title{
Uptake and barriers for implementation of the resect and discard strategy: an international survey
}

\section{(ㄷ)(우우}

Authors

Philippe Willems*, 1,2, Roupen Djinbachian*, 1,2, Saskia Ditisheim²,3, Sinan Orkut ${ }^{4}$, Heiko Pohl' ${ }^{5}$, Alan Barkun ${ }^{6}$, Mickael Bouin $^{2,3}$, Bernard Faulques ${ }^{2,3}$, Daniel von Renteln ${ }^{2,3}$

Institutions

1 Division of Internal Medicine, Montreal University Hospital Center (CHUM), Montreal, Canada

2 Montreal University Hospital Research Center (CRCHUM), Montreal, Canada.

3 Division of Gastroenterology, Montreal University Hospital Center (CHUM), Montreal, Canada

4 Faculty of Medicine, University of Strasbourg, Strasbourg, France

5 Department of Veterans Affairs Medical Center, White River Junction, Vermont, and Dartmouth Geisel School of Medicine, Hanover, New Hampshire, United States

6 Division of Gastroenterology, McGill University Health Center, McGill University, Montreal, Canada

submitted 21.11.2019

accepted after revision 4.2.2019

\section{Bibliography}

DOI https://doi.org/10.1055/a-1132-5371 |

Endoscopy International Open 2020; 08: E684-E692

(c) Georg Thieme Verlag KG Stuttgart · New York eISSN 2196-9736

\section{Corresponding author}

Daniel von Renteln, MD, Department of Medicine, Division of Gastroenterology, Montreal University Hospital (CHUM) and Montreal University Hospital Research Center (CRCHUM), 900 Rue Saint-Denis, Montréal, QC H2X 0A9, Montreal, Canada

Fax: +1-514-412-7287

renteln@gmx.net

丹 Supplementary material

Online content viewable at:

https://doi.org/10.1055/a-1132-5371

\section{ABSTRACT}

Background and study aims Optical real-time diagnosis (= resect-and-discard strategy) is an alternative to histopathology for diminutive colorectal polyps. However, clinical adoption of this approach seems sparse. We were interested in evaluating potential clinical uptake and barriers for implementation of this approach.

Methods We conducted an international survey using the "Google forms" platform. Nine endoscopy societies distributed the survey. Survey questions measured current clinical uptake and barriers for implementing the resect-and-discard strategy, perceived cancer risk associated with diminutive polyps and potential concerns with using CT-colonography as follow-up, as well as non-resection of diminutive polyps.

Results Eight hundred and eight endoscopists participated in the survey. $84.2 \%$ (95\% Cl 81.6\%-86.7\%) of endoscopists are currently not using the resect-and-discard strategy and $59.9 \%$ (95\% Cl 56.5\%-63.2\%) do not believe that the resect-and-discard strategy is feasible for implementation in its current form. European (38.5\%) and Asian (45\%) endoscopists had the highest rates of resect-and-discard practice, while Canadian (13.8\%) and American (5.1\%) endoscopists had some of the lowest implementation rates. $80.3 \%$ (95\% Cl 77.5\%-83.0\%) of endoscopists believe that using the resect-and-discard strategy for diminutive polyps will not increase cancer risk. 48.4\% (95\% Cl 45.0\%-51.9\%) of endoscopists believe that leaving diminutive polyps in place is associated with increased cancer risk. This proportion was slightly higher (54.7\%; 95\% Cl 53.6\%-60.4\%) when asked if current CT-colonography screening practice might increase cancer risks.

Conclusion Clinical uptake of resect-and-discard is very low. Most endoscopists believe that resect-and-discard is not feasible for clinical implementation in its current form. The most important barriers for implementation are fear of making an incorrect diagnosis, assigning incorrect surveillance intervals and medico-legal consequences.

\footnotetext{
* These authors contributed equally.
} 


\section{Introduction}

Diminutive polyps ( $\leq 5 \mathrm{~mm}$ ) are the most common types found during colonoscopies [1]. Their histopathologic examination is costly while their potential for malignancy is low [1-3]. Because diminutive polyps have such a low risk for harboring or progressing to cancer it might be reasonable to forgo histopathology in favor of less costly strategies. Image enhanced endoscopy can be used for real-time optical diagnosis of colorectal polyps. Validated classification systems based on color, vascular pattern and other criteria have been developed to determine if a polyp is neoplastic or not. The approach of replacing histopathology with optical diagnosis has been named the resect-and-discard strategy [4-7]. Studies have shown that in the hands of adequately trained endoscopists, real-time optical diagnosis provides a good alternative to pathology while being more cost-effective $[8,9]$. Consequently, multiple gastroenterology societies have recommended adopting resect-and-discard as part of screening programs if certain quality standards can be met [10-12]. Clinical implementation of this approach, however, seems sparse.

We were therefore interested in evaluating the current clinical uptake and barriers for implementation of the resect-anddiscard strategy, understanding the perceived cancer risk for diminutive polyps among endoscopists and potential concerns with leaving diminutive polyps unresected or with using computed tomography-colonography in screening and surveillance.

\section{Methods}

The Checklist for Reporting Results of Internet E-Surveys (CHERRIES) criteria was used to present the results of our survey [13].

\section{Study design}

A cross-sectional international survey study was conducted. Target population were endoscopists with active colonoscopy practice regardless of profession, case-load, or experience. The survey was developed through discussion and consensus between 3 gastroenterologists (H.P., A.B., D.vR.). The study protocol and survey were approved by the CRCHUM Institutional Review Board (CER-number: 17.063). No personal information was collected or stored during the survey.

\section{Recruitment process}

Twenty-nine Gastroenterology, Endoscopy and Surgical associations were contacted between July 2017 and May 2018 (Table 51 ). These associations were asked to send our online survey to their members using their mailing list, newsletter or social media (Twitter and Facebook). Survey participation was on a voluntary basis. The survey was advertised as taking a total of 3 minutes to complete. No monetary or non-monetary incentives were provided for the completion of our survey. Double clicking was allowed, results were only transmitted to the database if the participant clicked on survey completed at the end of the questionnaire. The survey advertisement text is available in Appendix 1.

\section{Survey content}

We used "Google Forms" to administer an online questionnaire comprising 26 questions distributed on five pages (Appendix 2). The questions were listed in the same order for all participants. Participants were able to return to previous pages to modify their answers. The survey was only accessible through the link provided to the contacted associations.

The primary outcome was endoscopist uptake of the resectand-discard strategy in current clinical practice. Secondary outcomes included perceived feasibility and barriers to implementation for a resect-and-discard strategy; perceived cancer risk for resect-and-discard implementation; perceived cancer risk for diminutive polyps; concerns with leaving diminutive polyps unresected; and the perceived cancer risk of adopting computed tomography $(\mathrm{CT})$ colonography as primary screening and surveillance strategy.

The survey collected information on participant demographics and practice setting data including the country of practice; private, academic or mixed practice setting; training specialty; years in practice; number of yearly colonoscopies performed and practice reimbursement. The survey further assessed participants' knowledge of the existence of the resectand-discard strategy; current practice implementation and barriers for implementation of a resect-and-discard approach; opinion on cancer risk associated with diminutive polyps and the resect-and-discard strategy; as well as usage of forceps/snares for different polyp types. The survey questions with possible answer options can be found in Appendix 2.

\section{Statistical analysis}

We used descriptive analysis with frequency and percentages to describe the participant's characteristic and their answers. To examine the association between participant characteristics and survey responses, we used univariate using chi square test and multivariate analysis using multilevel logistic regression model. A two-tailed $P<0.05$ was considered significant. For statistical analysis, SPSS 25 (Chicago, Illinois, United States) was used.

\section{Results}

The survey was distributed by nine associations (Table S2). We estimated that a total of 13,818 individuals were reached through emails based on the feedback we received regarding societies' mailing lists. An additional 8,991 individuals were reached through social media. This number was estimated by the number of unique views from our posts on social media pages or the number of people following the page at the time of the post if the number of unique views was not accessible. This resulted in a distribution to 21,807 individuals, not considering individuals that could be members of more than one association. Overall, 808 responders participated in the survey. The response rate was $3.7 \%$. Most of the responses received were from the ACG mailing list (459/808 responders). Response rate from ACG members was $3.8 \%$, which was similar to the overall 
- Table 1 Characteristic of survey participants.

\begin{tabular}{|c|c|}
\hline Characteristics & Responses $(n=808)$ \\
\hline Country of practice & $\mathbf{N}(\%)$ \\
\hline - United States of America & $454(56.2)$ \\
\hline - Europe & $92(11.4)$ \\
\hline - Canada & $80(9.9)$ \\
\hline - Asia & $61(7.5)$ \\
\hline - South/Central America & $55(6.8)$ \\
\hline - Australia/New Zealand & $29(3.6)$ \\
\hline - Other & $25(3.1)$ \\
\hline - Missing & $12(1.5)$ \\
\hline \multicolumn{2}{|l|}{ Practice setting } \\
\hline - Private & $308(38.1)$ \\
\hline - Academic & $233(28.8)$ \\
\hline - Community Hospital & $133(16.5)$ \\
\hline - Mixed & $126(15.6)$ \\
\hline - Missing & $8(1.0)$ \\
\hline \multicolumn{2}{|l|}{ Training and level } \\
\hline - Gastroenterologist & $682(84.4)$ \\
\hline - Resident/Fellow in training & $50(6.2)$ \\
\hline - General Surgeon & $38(4.7)$ \\
\hline - Colorectal Surgeon & $14(1.7)$ \\
\hline - Internal Medicine & $10(1.2)$ \\
\hline - Nurse Endoscopist & $6(0.7)$ \\
\hline - Missing & $8(1.0)$ \\
\hline \multicolumn{2}{|l|}{ Years in practice } \\
\hline - Less than 10 years & $373(46.2)$ \\
\hline - Between 10-20 years & $166(20.5)$ \\
\hline - More than 20 years & $258(31.9)$ \\
\hline - Missing & $11(1.4)$ \\
\hline \multicolumn{2}{|l|}{ Colonoscopies performed each year } \\
\hline - Less than 100 & $33(4.1)$ \\
\hline . $100-300$ & $151(18.7)$ \\
\hline . 301-500 & $163(20.2)$ \\
\hline - More than 500 & $454(56.2)$ \\
\hline - Missing & $7(0.9)$ \\
\hline \multicolumn{2}{|l|}{ Practice reimbursement } \\
\hline - Fee per colonoscopy procedure & $368(45.5)$ \\
\hline - Salary & $266(32.9)$ \\
\hline - Mixed & $165(20.4)$ \\
\hline - Missing & $9(1.1)$ \\
\hline
\end{tabular}

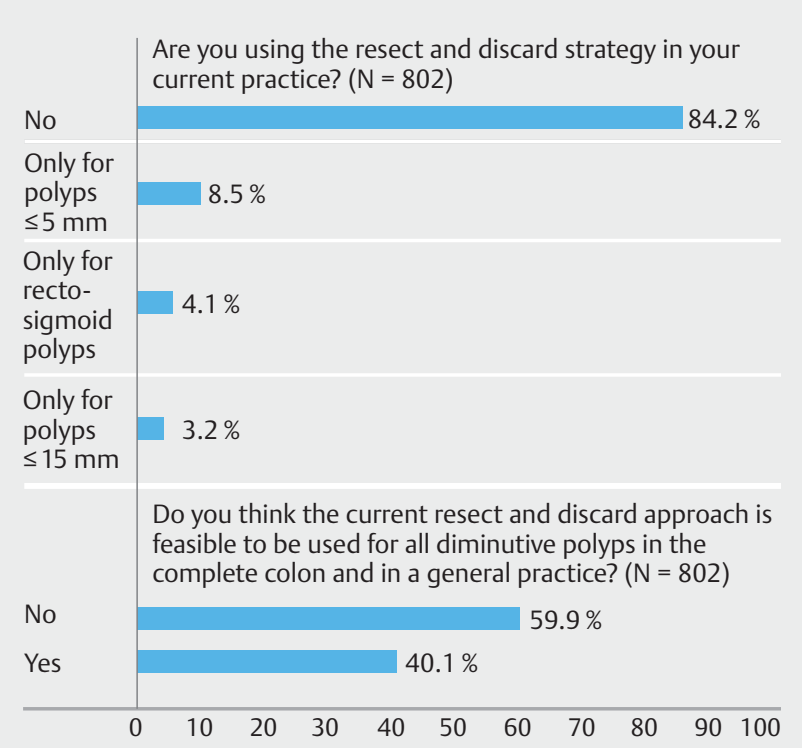

Fig. 1 Endoscopist usage of resect-and-discard strategy and perceptions of feasibility.

response rate of $3.7 \%$ using newsletter, mailing list and social medias $(P=0.96)$.

\section{Endoscopist demographics}

The majority of survey participants were from to the United States (56.2\%), Europe (11.4\%) and Canada (9.9\%). Most participants were Gastroenterologists (84.4\%). Endoscopist practice setting was private $(38.1 \%)$, academic $(28.8 \%)$, and community-based (16.5\%). $45.5 \%$ of endoscopists were reimbursed on a fee-for-service and $32.9 \%$ on a salary basis. More details provided in $>$ Table 1 .

\section{Attitudes and practices with regards to a resect-and-discard strategy}

$84.2 \%$ (95\% Cl $81.6 \%-86.7 \%$ ) of endoscopists were not using the resect-and-discard strategy at the time of the survey, while $59.9 \%$ (95\% Cl 56.5\%-63.2\%) did not believe such an approach was feasible for implementation (> Fig. 1). However, $80.3 \%$ (95\% Cl $77.5-83.0 \%$ ) of endoscopists believed that using a resect-and-discard strategy for diminutive polyps would not lead to an increased cancer risk for patients. Practicing in Canada $(\mathrm{OR}=0.25 ; 95 \% \mathrm{Cl} 0.12-0.51)$ and in the United States $(\mathrm{OR}=0.09 ; 95 \% \mathrm{Cl} 0.05-0.14)$ were factors that were statistically significantly associated with not practicing a resect-and-discard strategy in the multivariate analysis ( $>$ Table 2 ).

When stratified by region, the majority of endoscopists (range: 75.4-90.1\%) replied that they had heard of the resectand-discard strategy, however, most were not using it in their current practice (range: 55-94.9\%). European (38.5\%) and Asian (45\%) endoscopists had the highest rates of resect-anddiscard implementation, while Canadian (13.8\%) and American (5.1\%) endoscopists displayed lower rates. When asked if this strategy would be feasible in general practice most endos- 
- Table 2 Comparison of demographic characteristics between survey participants who use resect-and-discard in their current practice and those who don't.

\begin{tabular}{|c|c|c|c|c|}
\hline Use of the resect-and-discard & $\operatorname{Yes}^{1} \mathrm{n},(\%)$ & No $n,(\%)$ & univariate analysis & multivariate analysis (OR) \\
\hline Country of practice & & & 0.01 & \\
\hline - Australia/New Zealand & $5(17.2 \%)$ & $24(82.7 \%)$ & & n.s. \\
\hline - Canada & $11(13.7 \%)$ & $69(86.3 \%)$ & & $0.25(0.12 ; 0.51)$ \\
\hline - United States & $23(5.1 \%)$ & $431(94.9 \%)$ & & $0.09(0.05 ; 0.14)$ \\
\hline - Asia & $27(45.0 \%)$ & $33(55.0 \%)$ & & n.s. \\
\hline - Europe & $35(38.5 \%)$ & $56(61.5 \%)$ & & n.s. \\
\hline - South/Central America & $13(23.6 \%)$ & $42(76.4 \%)$ & & n.s. \\
\hline - Other & $9(44.0 \%)$ & $14(56.0 \%)$ & & n.s. \\
\hline Practice setting & & & 0.40 & \\
\hline - Academic & $39(16.7 \%)$ & $194(83.3 \%)$ & & n.s. \\
\hline - Community hospital & $23(17.4 \%)$ & $109(82.6 \%)$ & & n.s. \\
\hline - Private & $41(13.3 \%)$ & $267(86.7 \%)$ & & n.s. \\
\hline - Mixed & $24(18.4 \%)$ & $102(81.6 \%)$ & & n.s. \\
\hline Training and level & & & 0.30 & \\
\hline - Gastroenterologist & $104(15.3 \%)$ & $577(84.7 \%)$ & & n.s. \\
\hline - Colorectal surgeon & $4(28.6 \%)$ & $10(71.4 \%)$ & & n.s. \\
\hline - General surgeon & $8(21.6 \%)$ & $29(78.4 \%)$ & & n.s. \\
\hline - Internal medicine & $3(30.0 \%)$ & $7(70.0 \%)$ & & n.s. \\
\hline - Nurse endoscopist & $1(16.7 \%)$ & $5(83.3 \%)$ & & n.s. \\
\hline - Resident/fellow & $7(14.0 \%)$ & $43(86.0 \%)$ & & n.s. \\
\hline Years in practice & & & 0.01 & \\
\hline - $<10$ & $72(19.4 \%)$ & $300(80.6 \%)$ & & n.s. \\
\hline - $10-20$ & $23(13.9 \%)$ & $142(86.1 \%)$ & & n.s. \\
\hline.$>20$ & $30(11.6 \%)$ & $228(88.4 \%)$ & & n.s. \\
\hline Colonoscopies per year & & & $<0.01$ & \\
\hline - $<100$ & $12(36.4 \%)$ & $21(63.6 \%)$ & & n.s. \\
\hline - 100-300 & $32(21.2 \%)$ & $119(78.8 \%)$ & & n.s. \\
\hline " 301-500 & $26(16.0 \%)$ & $136(84.0 \%)$ & & n.s. \\
\hline . $>500$ & $57(12.6 \%)$ & $396(87.4 \%)$ & & n.s. \\
\hline Procedure reimbursement & & & 0.01 & \\
\hline - Fee per procedure & $45(12.2 \%)$ & $323(87.8 \%)$ & & n.s. \\
\hline - Salary & $47(17.7 \%)$ & $219(82.3 \%)$ & & n.s. \\
\hline - Mixed & $34(20.9 \%)$ & $129(79.1 \%)$ & & n.s. \\
\hline
\end{tabular}




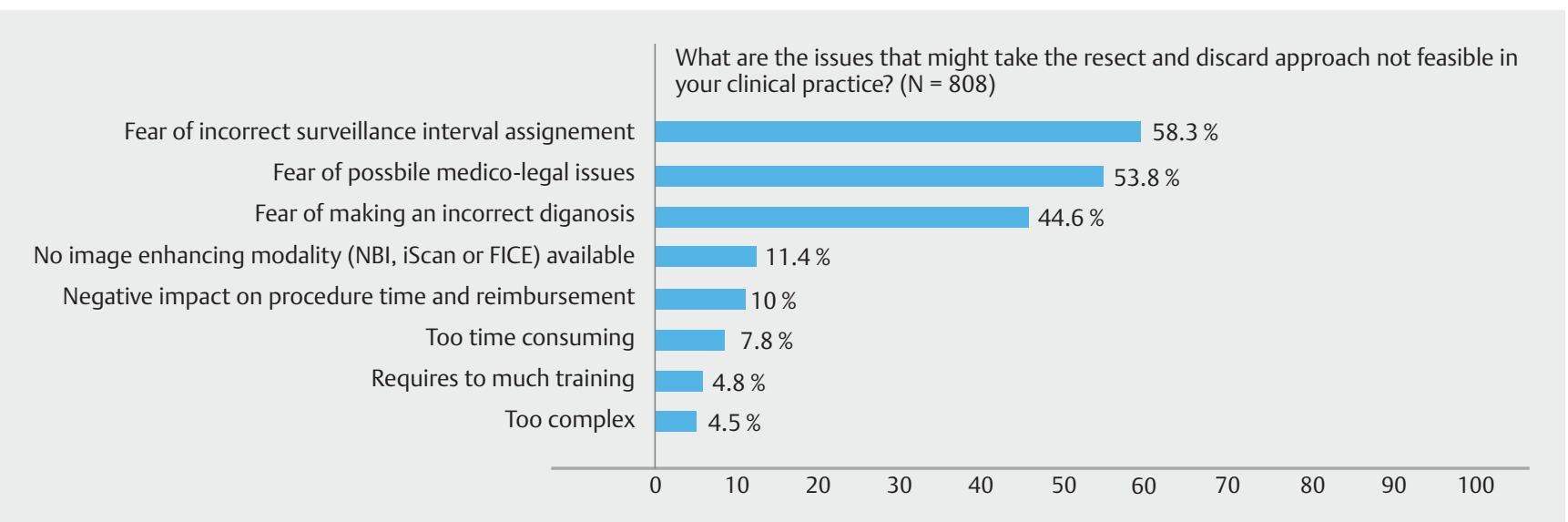

Fig. 2 Endoscopist perception of reasons for resect-and-discard non-feasibility. Multiple answers were allowed.

copists replied "no" with the exception of European endoscopists, $54.3 \%$ of whom replied "yes". There was geographic agreement that resect-and-discard did not increase patient cancer risk (Table S3).

When asked about barriers for implementation of a resectand-discard strategy $44.6 \%$ of participants $(95 \% \mathrm{Cl} 41.1-$ $48.0 \%$ ) were afraid of making a wrong diagnosis, $53.8 \%$ (95\% Cl 50.4-57.3\%) were concerned of potential medico-legal issues and $58.3 \%$ (95\% Cl 54.9-61.7\%) were afraid of assigning incorrect surveillance intervals to patients ( $>$ Fig. 2). For American endoscopists, the most important issue preventing implementation of a resect-and-discard strategy was fear of medicolegal issues (67.2\%), which was significantly more than the rest of the world ( $37.7 \%$; $P$ 0.001). Fear of making a wrong diagnosis was the main barrier for the majority of endoscopists from Australia/New Zealand (60\%) and Asia (37.7\%). For the rest of the world, fear of incorrect surveillance interval assignment was the most important issue (Table S4).

\section{Perception of cancer risk associated with diminutive polyps}

Overall, $63.0 \%$ of survey participants partly or completely agreed that diminutive polyps can be left unresected until the next screening colonoscopy because of a low associated cancer risk. Endoscopists were however evenly split on the effects of leaving such polyps unresected with regards to patient cancer risk, with $48.4 \%$ (95\% Cl 45.0\%-51.9\%) thinking that leaving diminutive polyps in place would increase the cancer risk of patients ( $>$ Table 3 ).

When stratified by region, only $20 \%$ of participants from Australia/New Zealand partly or completely agreed that diminutive polyps can be left unresected until the next screening colonoscopy, in contrast to participants from all other regions where between 58.2 and $68 \%$ partly or completely agreed that this approach was safe. The majority of endoscopists from the United States (51.3\%), Asia (59.0\%) and South/central America (54.5\%) thought that leaving diminutive polyps in place could increase cancer risk. In contrast, most European (58.2\%) and
Canadian (63.3\%) endoscopists thought that this was not so (Table S5).

There was no consensus on appropriate follow-up after leaving diminutive polyps in place. $61.1 \%$ of North American endoscopists chose a maximum of 5-year follow-up after unresected diminutive polyps compared to other regions where $74.4 \%$ chose a 3-year or less maximum follow-up $(P<0.001)$. Endoscopists from the United States (12.5\%) and Canada (10.1\%) had much higher rates of recommending the maximum option of 10-year follow-up after unresected diminutive polyps. When stratified by profession (Gastroenterologist, surgeon, internist, nurse endoscopist), $50.3 \%$ of gastroenterologists chose a maximum of 5 -year follow-up and $73.5 \%$ of other specialties chose a maximum of 3-years or less follow-up when leaving diminutive polyps unresected $(P=0.003)$.

\section{Perceptions of non-resection of diminutive polyps and use of CT-Colonography}

Overall, $52 \%$ of endoscopists were leaving non-adenomatous appearing diminutive polyps in place in their current practice. Geographical distribution was very variable: between $37.7 \%$ (Asia) and $80.0 \%$ (Australia/ New Zealand) of endoscopists responded that they were leaving non-adenomatous appearing diminutive polyps in place in their current practice (Table S5).

Of the surveyed endoscopists, 54.7\% (95\% Cl 53.6-60.4\%) thought that current CT-colonography guidelines were "probably" or "definitely" putting patients at higher cancer risk ( $\triangleright$ Table 4). When stratified by region, the majority of American, European, Asian and South/Central American endoscopists agreed with that statement versus a minority of Canadian (32.5\%) and Australian/New Zealand (20\%) endoscopists.

\section{Discussion}

To our knowledge, our study represents the largest international survey available to date studying endoscopist opinion on resect-and-discard and diminutive polyps. It is also the first providing data on geographic differences in endoscopist attitudes 
- Table 3 Endoscopist perceptions on the cancer risk of diminutive polyps.

\section{Questions $^{1}$}

Responses; N (\%)

Do you think that leaving diminutive polyps increases the risk of cancer of patients?

$N=797$

$\begin{array}{ll}\text { No } & 411\end{array}$

(51.6)

Yes

386

$(48.4)$

Cancer risk in a diminutive polyp is so low that such polyp can be left unresected until the next follow-up colonoscopy

$\mathrm{N}=803$

Iagree

101

I partly agree

405

I partly disagree

129

I completely disagree

168

If you leave a diminutive polyp unresected, the next colonoscopy should be within a maximum of

$N=790$

1 year

92

3 years

245

5 years

383

10 years

70

Do you leave diminutive polyps (up to $5 \mathrm{~mm}$ ) in place in your current practice?

$\mathrm{N}=808$

Sometimes

370

In the majority of cases

51

Always

If appearance of the polyp suggests it is non-adenomatous

420

(52.0)

If patient is on anticoagulation medication

170

If patient has severe comorbidities

161

If follow-up colonoscopy already scheduled

${ }^{1}$ Multiple answers were allowed

- Table 4 Endoscopist perceptions of CT-colonography, resect-and-discard and cancer risk.

\section{Questions}

Do you think that current $\mathrm{CT}$-colonography practice, which leaves polyps $<6 \mathrm{~mm}$ in place until the next surveillance exam, leads to an increased risk of colon cancer for the patient?

\begin{tabular}{|c|c|c|}
\hline No & 59 & $(7.3)$ \\
\hline Probably Not & 305 & $(37.9)$ \\
\hline Probably Yes & 324 & $(40.3)$ \\
\hline Yes & 116 & $(14.4)$ \\
\hline Do you think that using the resect-and-discard strategy for diminutive polyps increase the risk of cancer of patients? & \multicolumn{2}{|c|}{$N=808$} \\
\hline No & 639 & $(80.3)$ \\
\hline Yes & 157 & $(19.7)$ \\
\hline
\end{tabular}

towards resect-and-discard. Endoscopist characteristics were also well distributed according to practice setting, practice reimbursement and years in practice which lends external validity to our data. Geographic distribution was skewed towards North
America with endoscopists from the United States, Europe and Canada providing the majority of responses.

Our survey found that only $15.8 \%$ of endoscopists use the resect-and-discard strategy in their current practice and $59.9 \%$ 
thought that implementation was not feasible of the resectand-discard strategy in its current form. The most important reasons why the resect-and-discard strategy was not feasible included fear of making an incorrect diagnosis leading to incorrect surveillance interval assignment and medicolegal issues. Our results were similar to those found by Soudagar et al. 2016, where medicolegal concerns were the main barrier for implementation of the resect-and-discard strategy for the 105 Gastroenterologists surveyed during a national conference in the United States [14]. These reasons seem to point towards a concern of potential interval CRCs when using a resect-and-discard strategy, however, $80.3 \%$ of endoscopists voiced the opinion that a resect-and-discard strategy would not increase CRC risk. This could be caused by endoscopists feeling that interval cancer, while not more frequent with the resect-and-discard strategy, would be more difficult to explain in a possible future medicolegal pursuit. Very few endoscopists cited complexity of resect-and-discard and training requirements as barriers for implementation. While the consensus for most regions is that resect-and-discard was not feasible, European endoscopists showed an increased adoption of the strategy (54.3\%). Endoscopist practice can be dependent upon current healthcare culture, such as fear of medico-legal issues, acquiring new technology, and early adoption of new trends, which potentially explains the varying dispositions observed between geographic regions.

While the survey found that endoscopists did not completely trust their capacity to make accurate diagnoses, recent metaanalyses have shown that adequately trained endoscopists can achieve $>90 \%$ concordance with histology-based diagnosis and $>90 \%$ negative predictive value during optical diagnosis $[8,9]$. The American Society for Gastrointestinal Endoscopy (ASGE) and the European Society of Gastrointestinal Endoscopy (ESGE) currently recommend the use of resect-and-discard if these thresholds can be met. However other studies have shown that endoscopists can be unable to reach these benchmarks even after optical diagnosis training $[10,15,16]$. Most gastroenterology and endoscopy societies across the world have not yet officially made statements on resect-and-discard in their guidelines because of this contradictory data, contributing to the low implementation rate we found in this study. Further, recent research in imaging technologies and optical diagnosis have led to the emergence of many optical diagnosisbased classification systems, each with their own criteria, which can be confusing and overwhelming for endoscopists [17]. Our survey shows a definite concern from endoscopists about the feasibility of making optical diagnoses and highlights the need for simplifying decision-making or removing the need for optical diagnosis altogether [18]. A recent study has proposed a simplified and/or location-based strategy to reduce the need for optical diagnosis [19]. These simplified strategies achieved $>90 \%$ surveillance interval agreement compared to pathology and allowed for providing more patients with surveillance interval planning on the same day as the colonoscopy [19]. Other recent studies have proposed using only number and size of polyps as criteria for surveillance interval assignment $[20,21]$. This approach was able to achieve a $90 \%$ surveillance interval agreement with pathology in one instance and an $89.3 \%$ agreement in another $[20,21]$. More than $80 \%$ of patients could be provided with a surveillance interval on the same day using such an approach $[20,21]$. These alternative approaches thus appear promising even though endoscopists tended to prefer shorter intervals [20]. Limitations of simplified polyp (number and size only) or location-based strategies include the problem of not being able to distinguish between early signs of cancer (i. e. NICE-3 morphology) in optical diagnosis.

There was no consensus between endoscopists on cancer risk of diminutive polyps, with about $50 \%$ believing that leaving them unresected increased cancer risk. This result differs from a survey by Gellad et al. 2013, which reported that the majority of respondents would be somewhat agreeable to leave diminutive polyps in place if guidelines would support this practice [22]. Half of endoscopists in our study believed that CT-colonography increased patient risk. Studies on CT-colonography performance show a sensitivity of $>90 \%$ for the detection of polyps $>10 \mathrm{~mm}$ and $>95 \%$ for the detection of colorectal cancer [2325]. CT-colonography was, however, shown to have lower detection of high-risk SSAs when compared to colonoscopy [26] and exhibits poor sensitivity in diminutive polyp detection [27]. However, risk of malignancy of diminutive colon polyps was shown to be extremely low: $2 \%$ having advanced histology, and approximately $0.05 \%$ containing high grade dysplasia or neoplasia [1,2]. Studies with large numbers of small and diminutive polyps found no CRC present in any of these polyps $[2,28]$. Recent studies on the natural history of diminutive polyps showed a very indolent course for these polyps on follow-ups $[29,30]$. Current literature therefore suggests that risk of CRC arising from diminutive polyps is extremely low.

It is interesting to note that most endoscopists in our survey reported leaving polyps unresected in their patients and assigning 3- to 5-year maximum surveillance intervals when doing so. A recent meta-analysis has shown poor worldwide adherence for post-colonoscopy surveillance intervals [31]. Because resect-and-discard allows for providing more patients on the same day as the colonoscopy with a decision on when the next surveillance interval can be scheduled, clinical introduction of resect-and-discard might not only reduce immediate pathology costs but also upstream costs through avoiding unnecessary short follow-up surveillance intervals.

One important study limitation is the low response rate causing a potential for selection bias. However, since endoscopists can be members of multiple societies our response rate might be underestimated and represented a worst-case scenario. The advertising of our survey through Facebook/Twitter could also have skewed our sample towards younger endoscopists with more of an online presence or encourage double clicking from some of the participants. Furthermore, survey study always raise the issue of possible participation bias where survey participants are more incline to be up to date with recent guidelines and recommendations. Even in this selected population, resect-and-discard uptake was very low; suggesting the true uptake in the general population is probably even lower. The majority of responses were from the United States, Canada and Europe, which potentially limits our interpretation 
and generalizability to other regions. The survey was only available in English, which could have led to selection bias for certain regions of the world and for anglophone participants. However, we present the largest survey available to date on the topic.

\section{Conclusion}

In conclusion, current uptake of resect-and-discard is very low $(15.8 \%)$ with most endoscopists agreeing that such strategies are not feasible. Fear of making the wrong diagnosis and potential medicolegal repercussions are cited amongst the main reasons for difficulty of implementation. The development of simplified resect-and-discard models will likely provide a solution to these barriers for implementing resect-and-discard in clinical practise.

\section{Acknowledgements}

The findings, statements, and views expressed are those of the authors and do not represent the views of the Department of Veterans Affairs or the United States Government.

\section{Competing interests}

Dr. von Renteln is supported by a "Fonds de Recherche du Québec Santé" career development award and has received research funding from ERBE, Ventage, Pendopharm and Pentax and is a consultant for Boston Scientific and Pendopharm.

\section{References}

[1] Lieberman D, Moravec M, Holub J et al. Polyp size and advanced histology in patients undergoing colonoscopy screening: implications for CT colonography. Gastroenterology 2008; 135: 1100-1105

[2] Gupta N, Bansal A, Rao D et al. Prevalence of advanced histological features in diminutive and small colon polyps. Gastrointest Endosc 2012; 75: 1022-1030

[3] Kessler WR, Imperiale TF, Klein RW et al. A quantitative assessment of the risks and cost savings of forgoing histologic examination of diminutive polyps. Endoscopy 2011; 43: 683-691

[4] IJspeert JE, Bastiaansen BA, van Leerdam ME et al. Development and validation of the WASP classification system for optical diagnosis of adenomas, hyperplastic polyps and sessile serrated adenomas/ polyps. Gut 2016; 65: 963-970

[5] Hewett DG, Kaltenbach T, Sano Y et al. Validation of a simple classification system for endoscopic diagnosis of small colorectal polyps using narrow-band imaging. Gastroenterology 2012; 143: 599-607. e591

[6] lacucci M, Trovato C, Daperno M et al. Development and validation of the SIMPLE endoscopic classification of diminutive and small colorectal polyps. Endoscopy 2018; 50: 779-789

[7] Sano Y, Tanaka S, Kudo SE et al. Narrow-band imaging (NBI) magnifying endoscopic classification of colorectal tumors proposed by the Japan NBI Expert Team. Dig Endosc 2016; 28: 526-533

[8] McGill SK, Evangelou E, loannidis JP et al. Narrow band imaging to differentiate neoplastic and non-neoplastic colorectal polyps in real time: a meta-analysis of diagnostic operating characteristics. Gut 2013; 62: 1704-1713
[9] Abu Dayyeh BK, Thosani N, Konda V et al. ASGE Technology Committee systematic review and meta-analysis assessing the ASGE PIVI thresholds for adopting real-time endoscopic assessment of the histology of diminutive colorectal polyps. Gastrointest Endosc 2015; 81: 502 e $501-502$ e 516

[10] Rex DK, Kahi C, O'Brien M et al. The American Society for Gastrointestinal Endoscopy PIVI (Preservation and Incorporation of Valuable Endoscopic Innovations) on real-time endoscopic assessment of the histology of diminutive colorectal polyps. Gastrointest Endosc 2011; 73: 419-422

[11] Kaminski MF, Hassan C, Bisschops R et al. Advanced imaging for detection and differentiation of colorectal neoplasia: European Society of Gastrointestinal Endoscopy (ESGE) Guideline. Endoscopy 2014; 46: 435-449

[12] National Institute for Health Care and Excellence. Virtual chromoendoscopy to assess colorectal polyps during colonoscopy. Diagnostic Guidance [D628]. 2017: Available at (Accessed May 4th 2019): https://www.nice.org/uk/guidance/dg28

[13] Eysenbach G. Improving the quality of web surveys: the Checklist for Reporting Results of Internet E-Surveys (CHERRIES). J Med Internet Res 2004; 6: e34

[14] Soudagar AS, Nguyen M, Bhatia A et al. Are gastroenterologists willing to implement the "predict, resect, and discard" management strategy for diminutive colorectal polyps? results from a national survey J Clin Gastroenterol 2016; 50: e45-49

[15] McGill SK, Soetikno R, Rastogi A et al. Endoscopists can sustain high performance for the optical diagnosis of colorectal polyps following standardized and continued training. Endoscopy 2015; 47: 200-206

[16] Vleugels JLA, Dijkgraaf MGW, Hazewinkel Y et al. Effects of training and feedback on accuracy of predicting rectosigmoid neoplastic lesions and selection of surveillance intervals by endoscopists performing optical diagnosis of diminutive polyps. Gastroenterology 2018; 154: 1682-1693 e1681

[17] Djinbachian R, Dube AJ, von Renteln D. Optical diagnosis of colorectal polyps: recent developments. Curr Treat Options Gastroenterol 2019; 17: 99-114

[18] Atkinson NS, East JE. Optical biopsy and sessile serrated polyps: Is DISCARD dead? Long live DISCARD-lite! Gastrointest Endosc 2015; 82: $118-121$

[19] von Renteln D, Kaltenbach T, Rastogi A et al. Simplifying resect and discard strategies for real-time assessment of diminutive colorectal polyps. Clin Gastroenterol Hepatol 2018; 16: 706-714

[20] Hammar C, Frenn M, Pohl H et al. The polyp-based resect-and-discard strategy: a prospective study. Endoscopy 2019; 51: OP262

[21] Duong A, Bouin M, Leduc R et al. A7 The Polyp-based resect-and-discard strategy. J Can Assoc Gastroenterol 2019; 2: 15-16

[22] Gellad ZF, Voils Cl, Lin L et al. Clinical practice variation in the management of diminutive colorectal polyps: results of a national survey of gastroenterologists. Am J Gastroenterol 2013; 108: 873-878

[23] Johnson CD, Chen M-H, Toledano AY et al. Accuracy of CT colonography for detection of large adenomas and cancers. N Engl J Med 2008; 359: $1207-1217$

[24] Halligan S, Altman DG, Taylor SA et al. CT colonography in the detection of colorectal polyps and cancer: systematic review, Meta-analysis, and proposed minimum data set for study level reporting. Radiology 2005; 237: 893-904

[25] Pickhardt PJ, Hassan C, Halligan S et al. Colorectal cancer: CT colonography and colonoscopy for detection - systematic review and metaanalysis. Radiology 2011; 259: 393-405

[26] ljspeert JEG, Tutein Nolthenius C], Kuipers EJ et al. CT-colonography vs. colonoscopy for detection of high-risk sessile serrated polyps. Am J Gastroenterol 2016; 111: 516 
[27] Mulhall BP, Veerappan GR, Jackson JL. Meta-analysis: computed tomographic colonography. Ann Intern Med 2005; 142: 635-650

[28] Ponugoti PL, Cummings OW, Rex DK. Risk of cancer in small and diminutive colorectal polyps. Dig Liver Dis 2017; 49: 34-37

[29] Vleugels JLA, Hazewinkel Y, Fockens P et al. Natural history of diminutive and small colorectal polyps: a systematic literature review. Gastrointest Endosc 2017; 85: 1169-1176.e1161
[30] Vleugels JLA, Hassan C, Senore C et al. Diminutive polyps with advanced histologic features do not increase risk for metachronous advanced colon neoplasia. Gastroenterology 2019; 156: 623-634.e623

[31] Djinbachian R, Dubé A-J, Durand M et al. Adherence to post-polypectomy surveillance guidelines: a systematic review and meta-analysis. Endoscopy 2019; 51: 673-683 